\title{
Left Ventricle Postinfarction Pseudoaneurysm: Anatomical Forms and Surgical Management
}

\author{
J. M. Garrido ${ }^{*}$, A. Ferreiro², J. F. Rodríguez-Vázquez ${ }^{3}$, P. Prada², S. Verdugo ${ }^{3}$, J. Silva², \\ S. López-Checa ${ }^{4}$, I. Sánchez-Montesinos ${ }^{5}$ \\ ${ }^{1}$ Servicio de Cirugía Cardiovascular, HU Ramón y Cajal, Departamento de Ciencias Morfológicas, Universidad \\ Europea de Madrid, Madrid, Spain \\ ${ }^{2}$ Servicio de Cirugía Cardiovascular, HU Ramón y Cajal, Madrid, Spain \\ ${ }^{3}$ Departamento de Anatomía y Embriología Humana II, Facultad de Medicina, Universidad Complutense de \\ Madrid, Madrid, Spain \\ ${ }^{4}$ Servicio de Cirugía Cardiovascular, HU Virgen de las Nieves, Granada, Spain \\ ${ }^{5}$ Departamento de Anatomía y Embriología Humana, Facultad de Medicina, Universidad de Granada, Granada, Spain \\ Email: "josemanuel.garrido@salud.madrid.org, ${ }^{*}$ josemanuel.garrido@uem.es
}

Received 12 February 2014; revised 8 March 2014; accepted 15 March 2014

Copyright (C) 2014 by authors and Scientific Research Publishing Inc.

This work is licensed under the Creative Commons Attribution International License (CC BY). http://creativecommons.org/licenses/by/4.0/

(c) (i) Open Access

\section{Abstract}

Introduction: Left Ventricle Postinfarction Pseudoaneurysm (LVPS)-false aneurysm occurs after a free-wall rupture contained by the adjacent pericardium. LVPS lacks the normal structure of the ventricular wall and disrupts the normal chamber anatomy. However, the natural history, clinical presentation and surgical outcome are still unclear. For that reason, it is necessary to describe the most relevant anatomical characteristics of LVPS and the appropriate surgical strategies currently applied. Methods: We reviewed the anatomical characteristics of several patients diagnosed of LVPS and the surgical technique performed. In this work two different anatomical types of LVPS are described in detail, with the surgical and structural implications for left ventricle reconstruction. Results: There are two different anatomical forms of LVPS: 1) Typical pseudoaneurysm, with a small gateway neck between the Left Ventricle and the false aneurysm chamber (Figure 1(A)); 2) Atypical pseudoaneurysm, in which the anatomical defect is bigger, without well-defined edges, extends over a large segment of infarcted and thinned myocardial tissue. In both cases, the therapeutics targets and the surgical techniques used were directed to restore the normal geometry of Left Ventricle, keeping the optimal mitral valve function. Conclusions: The surgical key-step is to preserve or to remodel the ventricular chamber anatomy. This fact restores the ventricular geometry, not only removing the wall discontinuity that generated the pseudoaneurysm. Neverthe-

\footnotetext{
${ }^{*}$ Corresponding author.
}

How to cite this paper: Garrido, J.M., Ferreiro, A., Rodríguez-Vázquez, J.F., Prada, P., Verdugo, S., Silva, J., López-Checa, S. and Sánchez-Montesinos, I. (2014) Left Ventricle Postinfarction Pseudoaneurysm: Anatomical Forms and Surgical Management. Surgical Science, 5, 138-145. http://dx.doi.org/10.4236/ss.2014.54025 
less, final prognosis depends on the underlying ischemic cardiomyopathy and mechanical complications, such us mitral regurgitation or ventricular septal defect.

\title{
Keywords
}

\author{
Ventricular Anatomy, Left Ventricle Pseudoaneurysm Surgical Treatment, Ventricular \\ Remodelling, Restore of Left Ventricle, Morphology and Function of Left Ventricle, Ischemic \\ Cardiomyopathy
}

\section{Introduction}

Left ventricle (LV) is the principal systemic chamber of the heart and its anatomy is coupled to a high-pressure blood circuit physiology. LV wall presents an especial thickness due to its function [1]. Inside the chamber, we can distinguish two myocardial prominent points arranged in posterolateral and anteromedial position, which form the body of the papillary muscles. These muscular structures project multiple tendinous strings to the anterior and posterior mitral valve leaflets. Thereby, the normal mitral valve function depends on the ventricular integrity. According to Torrent-Guasp's theory of myocardial band, the shortening of muscular fibres produces the LV contraction, with the characteristic helicoidally torsion pattern (base to apex direction) [2]. The ventricular geometry and the anatomic preservation of this chamber are essential aspects to ensure the optimal cardiac function.

LV pseudoaneurysm (LVPS - false aneurysm occurs, in most cases, as a result of a transmural myocardial infarction. The wall thinning, secondary to an extensive myocardial necrosis, suffers a subsequent dilatation of the damaged tissue that progresses with each systole. This fact can finally produce a free-wall rupture, in a few cases contained by the adjacent pericardium, which allows the formation of the pseudoaneurysm. Therefore, LVPS lacks the normal structure of the ventricular wall, but it is only contained by the epicardium-pericardium reaction. However, the natural history, clinical presentation and surgical outcome are still unclear due to the rarity of occurrence and the small series of patient analyzed. Nevertheless, it seems to be clear that large LV pseudoaneurysm has a high risk of rupture and sudden death [3] [4]. We considerer that LV pseudoaneurysm should undergo surgical repair as soon as it is diagnosed, regardless of the patient's symptoms [5], maintaining the normal anatomy of LV chamber.

\section{Materials and Methods}

We reviewed the anatomical characteristics of several patients diagnosed of postinfarction left ventricle pseudoaneurysm (LVPS) and the surgical technique performed. To illustrate the surgical features of the anatomical types, we describe two cases well differenced. There are two different anatomical forms of LVPS: 1) Typical pseudoaneurysm, with a small gateway neck between the LV and the false aneurysm chamber (Figure 1(A)); 2) Atypical pseudoaneurysm, in which the anatomical defect is bigger, without well-defined edges, extends over a large segment of infarcted and thinned myocardial tissue (Figure 1(B)). Both types of LVPS lack the normal structure of a ventricular wall. The free-wall rupture generates an abnormal communication through the endocardium and myocardium, contained by the overlying pericardium. Therefore, LVPS differs from the true ventricular aneurysm (TVA) because the TVA is considered a pathological dilatation of the ventricle, although keeping the normal disposition of the wall layers (Figure 2).

In all cases the surgical goal was the comprehensive treatment of the cardiomyopathy, preserving the optimal ventricular geometry.

\section{Results}

\subsection{Typical Anatomical Form}

A 64-year-old man is an ex-smoker with hypercholesterolemia and arterial hypertension. The patient suffers an acute inferior myocardial infarction. The initial evolution was favourable, but 24 hours later, he began with chest pain and symptoms of pericarditis. Coronary angiography showed severe triple-vessel disease with right coronary ar- 

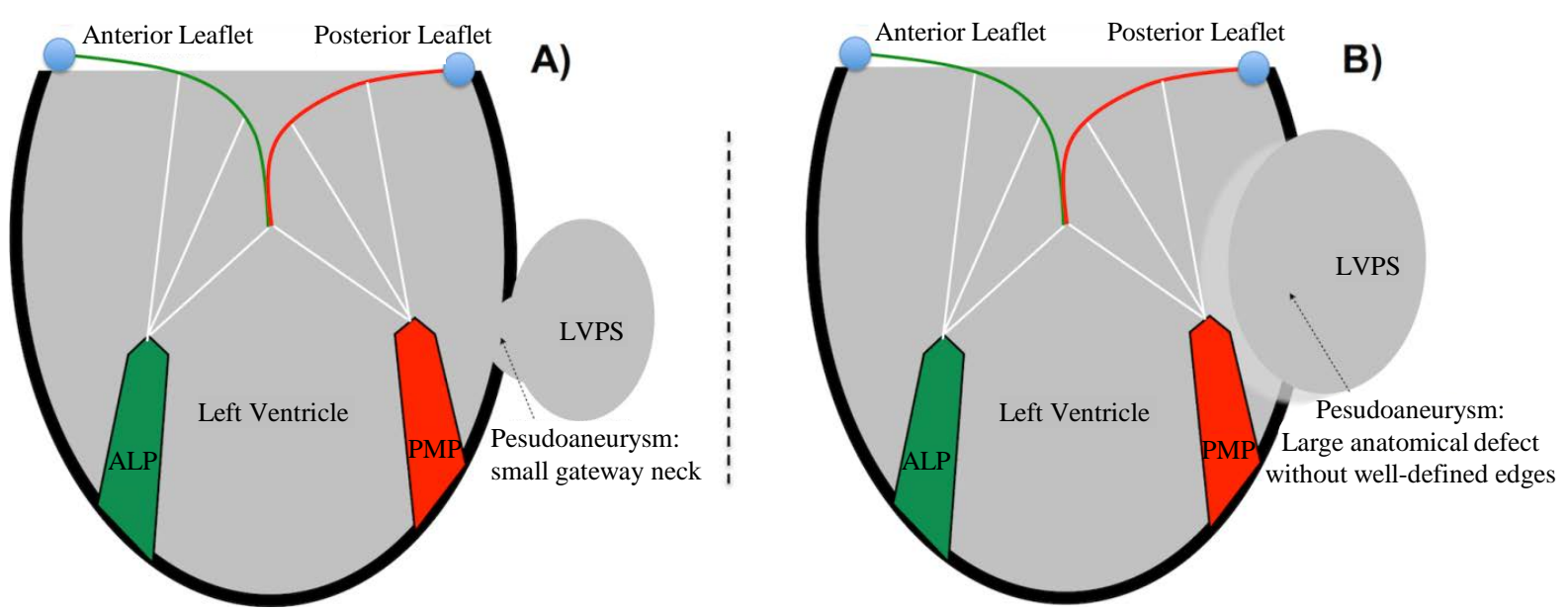

Figure 1. Schematic view of Left Ventricle Pseudoaneurysm (LVPS) anatomic forms. A) Typical anatomic form. Small gateway neck between LV and the pseudoaneurysm chamber. B) Atypical anatomic form. Extensive myocardial damage (wide opening to the abnormal chamber). ALP: antero-lateral papillary muscle. PMP: postero-medial papillary muscle.

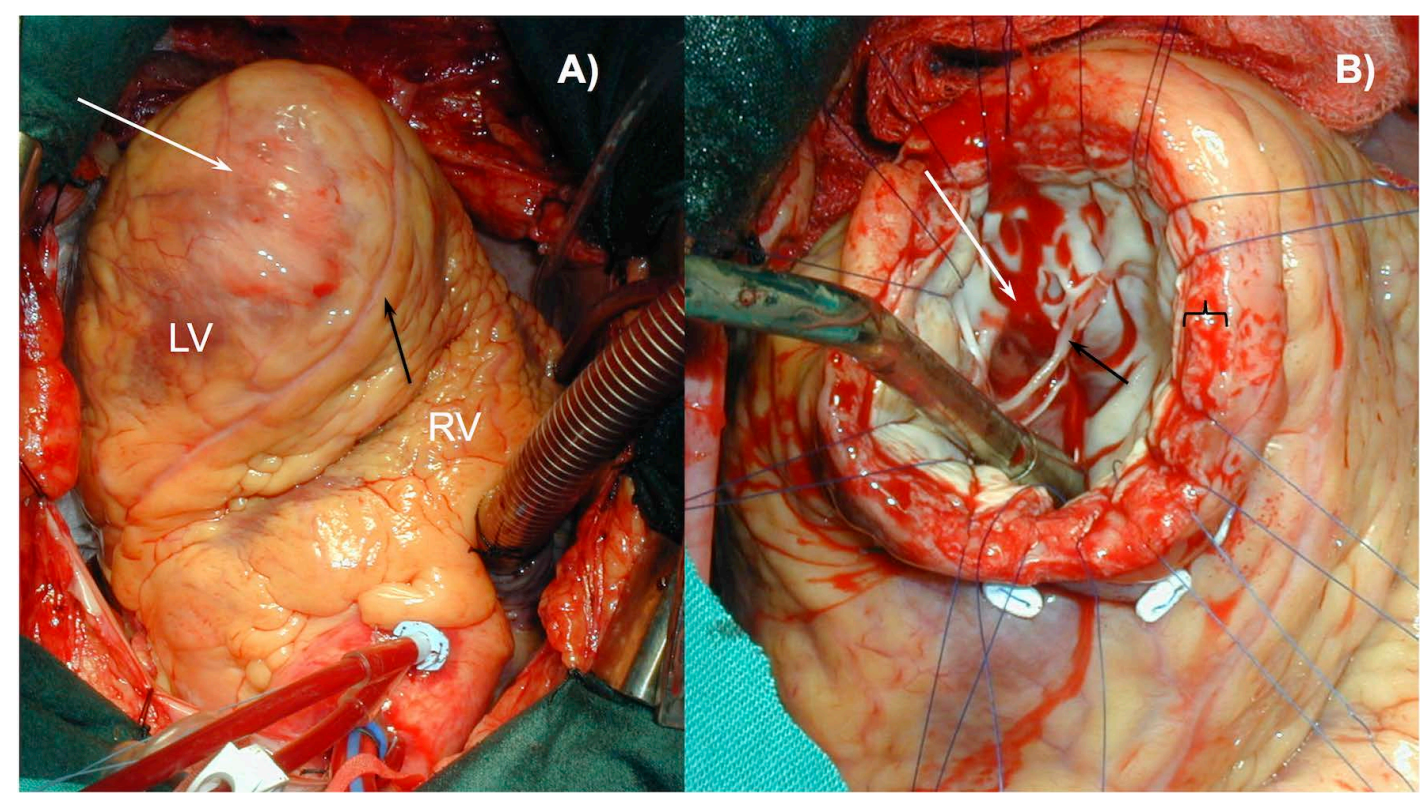

Figure 2. True Ventricular Aneurysm (TVA). A) Surgical view of an antero-apical TVA. The white arrow shows the external view of a giant TVA. The black arrow shows the anterior interventricular coronary artery (left anterior descending artery). B) Aneurysm chamber (white arrow). The black arrow shows mitral valve tendinae chordae. TVA maintains the normal wall architecture of left ventricle (black bracket).

tery occlusion. Contrast ventriculography (CV) showed the presence of a large inferior pseudoaneurysm. The diagnosis was confirmed by echocardiography, appreciating the characteristic hourglass image with a narrow neck between the left ventricle and the pseudoaneurysm chamber (Figure 3).

The patient was scheduled for surgery because of the high risk of pseudoaneurysm rupture and sudden death.

Under cardio-pulmonary bypass-CPB-(117 minutes), we separated the pseudoaneurysm from the adjacent pericardium. Subsequently, under cardiac arrest (86 minutes) we proceeded to open the chamber. Afterward, we identified the wall discontinuity of LV and decided to close the defect using a Dacron patch and fibrin sealants (Figure 4), without disrupt the normal ventricular geometry. This approach was used instead of a direct sutureline. In a second step we performed a complete myocardial revascularization. Postoperative, echocardiography showed no residual pseudoaneurysm with normal ventricular function. The patient made an uneventful recovery and was discharged on the ninth postoperative day. He was four days in the Intensive Care Unit. 


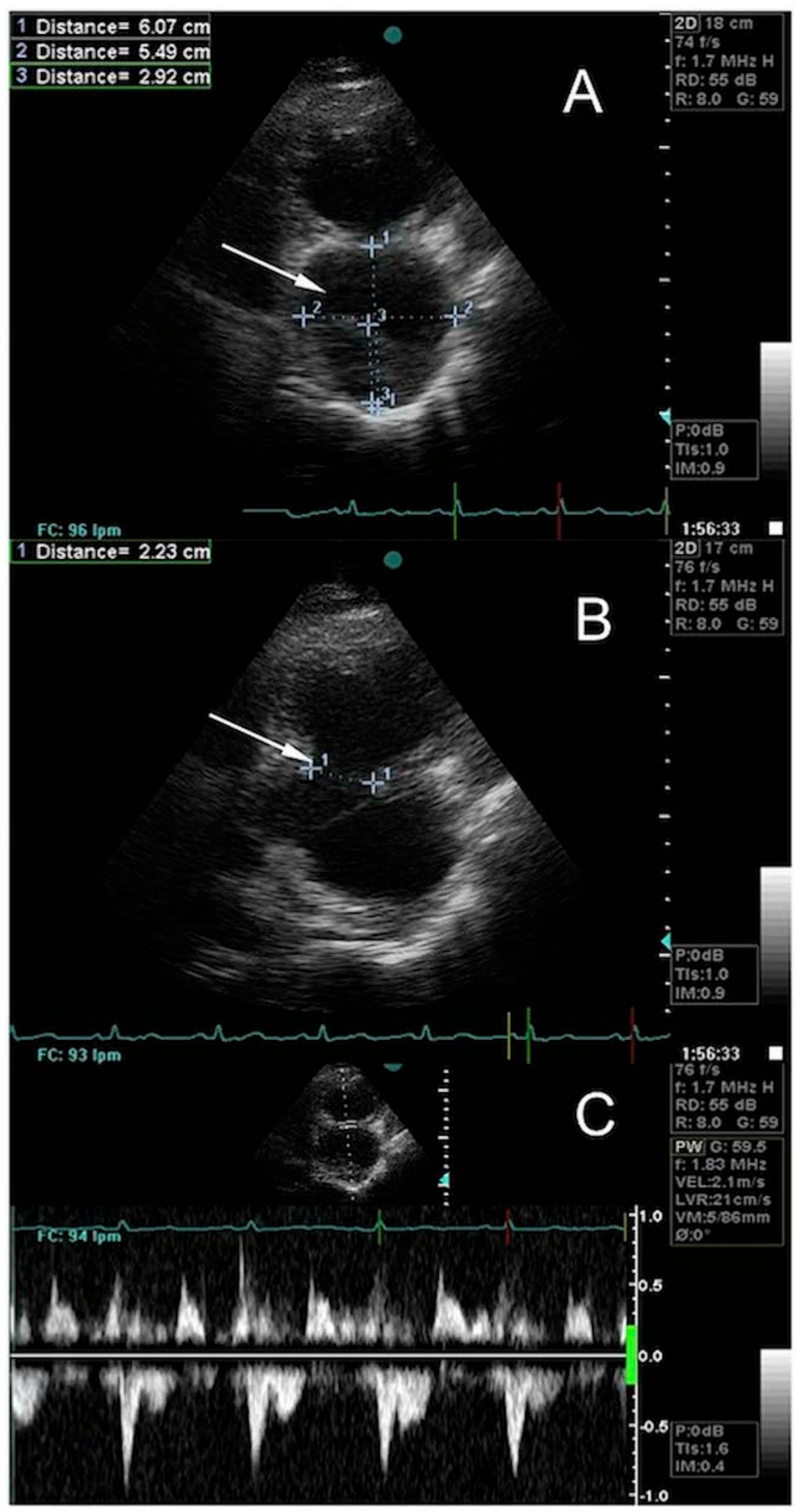

Figure 3. Echocardiographic view of a large left ventricle (LV) pseudoaneurysm. A) Characteristic hourglass image. The white arrow shows the pseudoaneurysm chamber. B) The white arrow shows the narrow neck between the LV and the pseudoaneurysm chamber. C) Blood flow between LV and the pseudoaneurysm. 


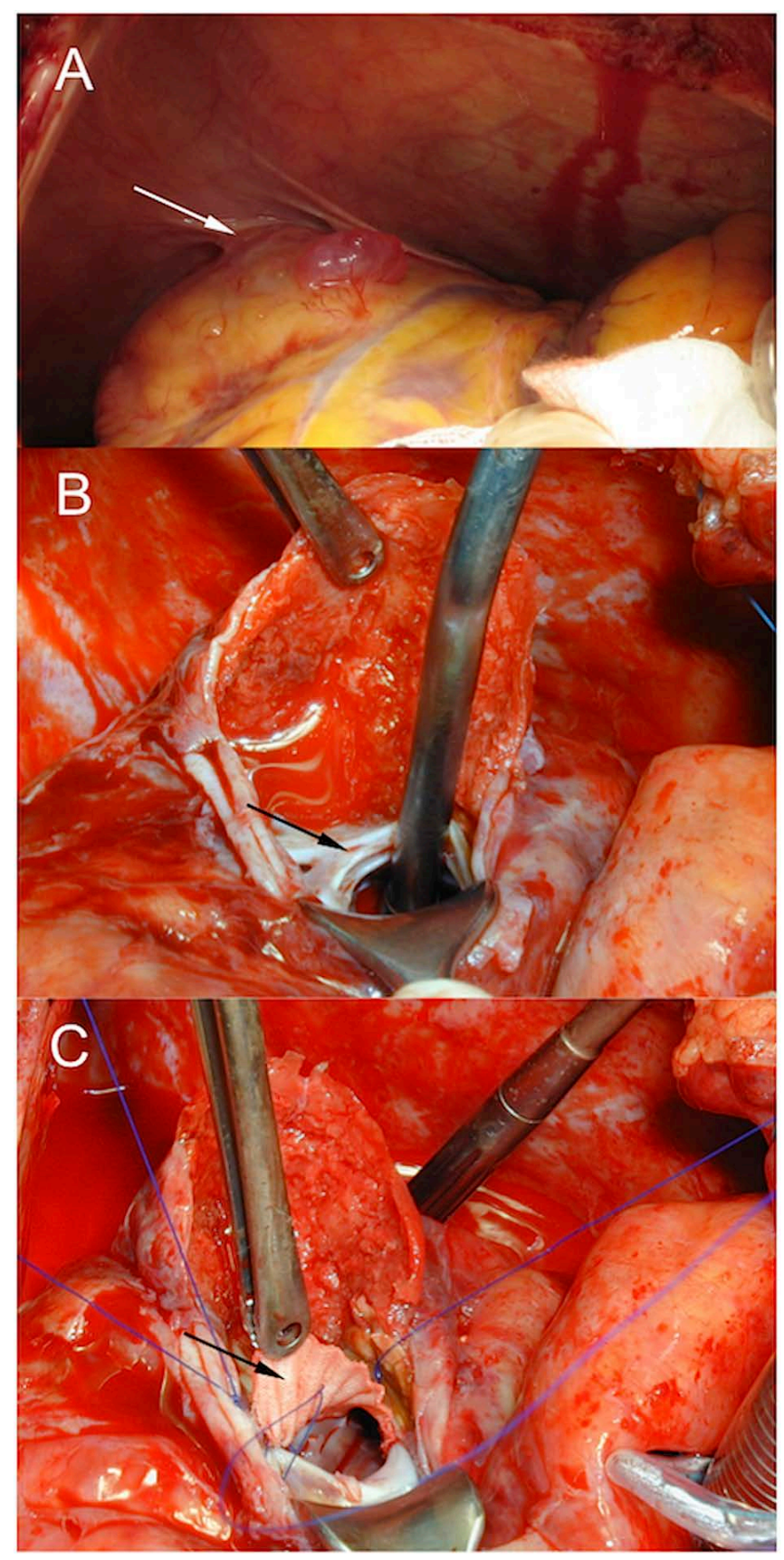

Figure 4. Surgical treatment of a posterior left ventricle pseudoaneurysm (Typical anatomic form). A) Inflammatory reaction of adjacent pericardium (white arrow). B) Open pseudoaneurysm without resecting the false aneurysm walls. The black arrow shows the neck between the LV and the pseudoaneurysm chamber. C) Patch repair of the pseudoaneurysm gateway (black arrow).

\subsection{Atypical Anatomic Form}

A 66-year-old man had a history of hypercholesterolemia, hypertension, ischemic cardiomyopathy and a prior inferior myocardial infarction in 1999. Since this first episode, he was diagnosed of non-revascularizable three-vessel disease with Left Ventricle Ejection Fraction (LVEF) of 25\% and an inferior ventricular aneurysm. Actually, the patient suffers a new acute myocardial infarction, discovering by echocardiography and contrast 
ventriculography (CV) the presence of a giant inferior pseudoaneurysm with worse ventricular function (Figure 5).

Due to the presence of heart failure, refractory to intensive pharmacological treatment, the patient underwent to surgery.

Under cardio-pulmonary bypass (CPB 123 minutes), without cardiac arrest, we separated the false aneurysm from the adjacent pericardium and performed a longitudinal incision of the pseudoaneurysm (Figure 5(A)). After identifying the limits of viable myocardium, we confirm the absence of a clear aneurysmal neck (Figure 5(B)). Therefore, we decided to restore the ventricular geometry using a wide PTFE patch, also reducing the ventricular volume, recovering the appropriate anatomy (Figure 5(C)). Afterward the pseudoaneurysm wall was closed over the patch with a double mattress suture and fibrin sealants.

Postoperative echocardiography showed no residual pseudoaneurysm (LVEF 37\%). The patient course was favourable and was discharged on the tenth postoperative day. He was two days in the Intensive Care Unit.

\section{Discussion}

The most frequent situation of postinfarction LV pseudoaneurysm is the posterior-inferior wall, because the acute rupture of the anterior or the lateral wall hardly ever is contended by the overlying pericardium [6]. We recommend opening the pericardium carefully due to the high risk of acute rupture. In all cases, the cardiac manipulation must be restricted to the indispensable areas to be effective for the CPB. Likewise, in the rare cases of anterior or lateral pseudoaneurysm, we prefer to use the femoral vessels before opening the chest.

When CPB is established, we need to separate the false aneurysm from the rest of pericardium tissue. Sometimes this issue is difficult because of the intense inflammatory reaction, otherwise essential to form the pseudoaneurysm. We consider that is not always necessary to induce cardiac arrest to repair the ventricular wall. So, in $\mathrm{CPB}$, we open longitudinally the pseudoaneurysm, avoiding the resection of its wall. Afterward we try to identify the pseudoaneurysm neck. In a typical LVPS, we found a narrow neck. Sometimes the gateway edges are consistent enough, with optimal fibrous tissue, to support a directly repair based on a suture-line [4] (Figure 6(A)). Although it is possible, we suggest an approach directed to preserve the normal ventricular geometry, particularly

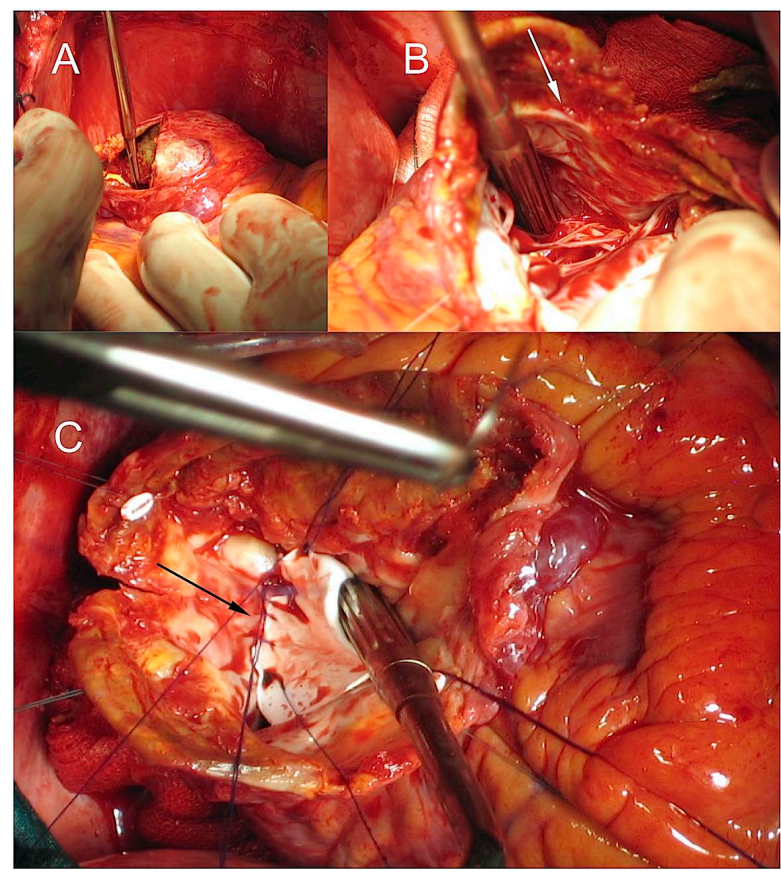

Figure 5. Surgical treatment of a posterior left ventricle pseudoaneurysm, generated on a non-viable myocardial aneurysmatic area (Atypical anatomic form). A) Open pseudoaneurysm without resecting the ventricular wall. B) Wide pseudoaneurysmal neck without clear anatomical limits (white arrow). C) Ventricular reconstruction with a chamber volume reduction using a PTFE patch (black arrow). 


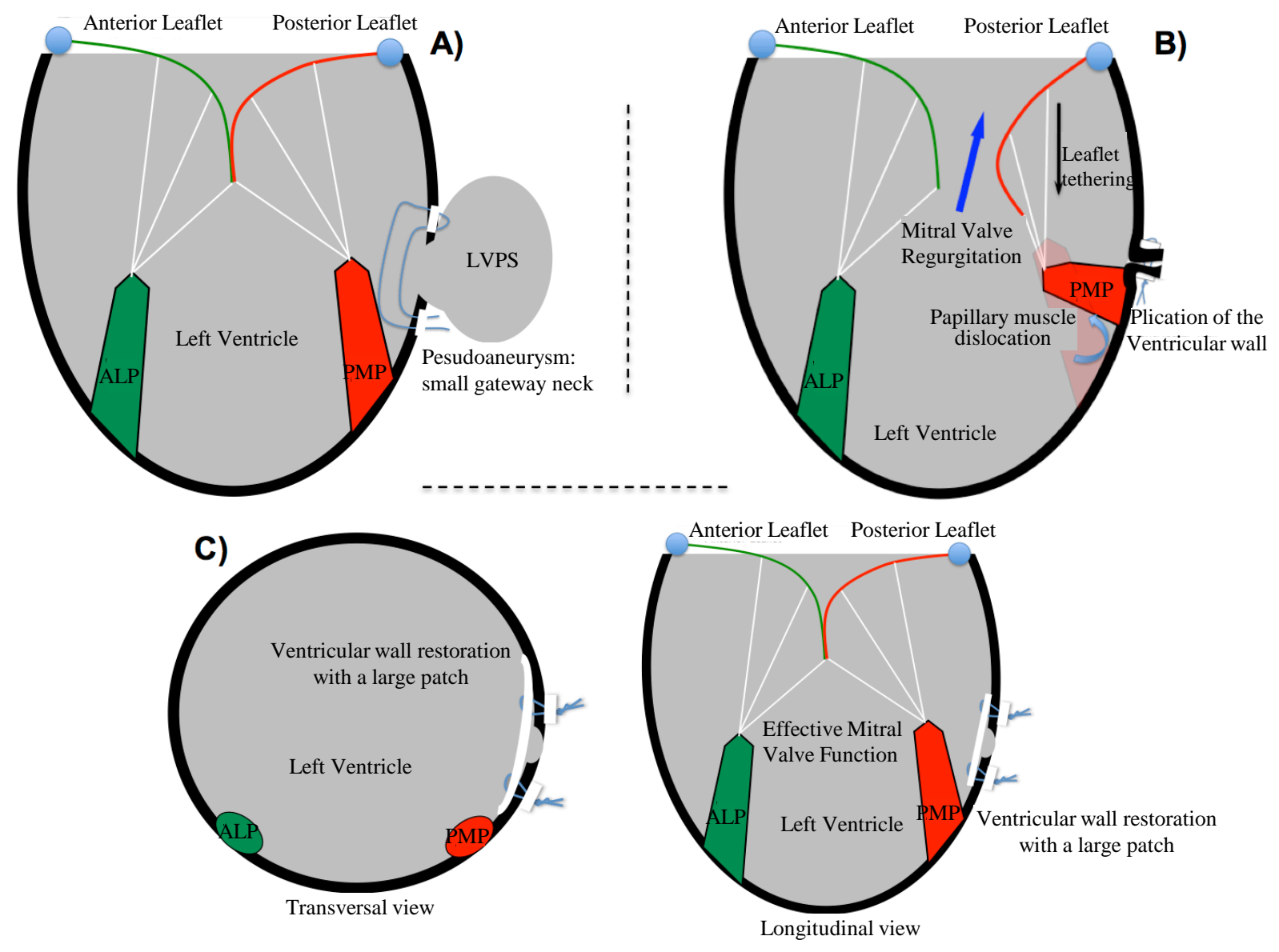

Figure 6. Schematic view of the surgical techniques used to correct Left Ventricle Pseudoaneurysm (LVPS). A) Direct suture line. B) The papillary muscle displacement produces mitral valve regurgitation. C) Repair of LVPS with a PTFE/ DACRON wide patch, restoring the normal anatomy of left ventricle. ALP: antero-lateral papillary muscle. PMP: posteromedial papillary muscle.

in posterior-inferior pseudoaneurysm. The direct repair in this location could modify, by the suture traction and the appropriate position of the papillary muscles, which could generate or increase mitral regurgitation (Figure 6(B)). Thus, we recommend a Dacron/PTFE wide patch in all cases (Figure 6(C)), avoiding the anatomy disruption and the papillary dislocation. This issue is especially important in pseudoaneurysms with a bigger neck, in which there is a lot of non-viable myocardial tissue [7] and it is necessary to restore the ventricular geometry. For that reason, the therapeutics targets and the surgical techniques used in TVA restoration are appropriated for the anatomical repair of the Left Ventricle Pseudoaneurysm.

\section{Conclusion}

The surgical goal is to preserve or to remodel the ventricular chamber anatomy. This fact normalizes the ventricular geometry, not only removing the wall discontinuity that generated the pseudoaneurysm. Then, it is useful to close the pseudoaneurysm walls over the patch and to use fibrin sealants between them to improve the haemostasis and the ventricular strain resistance. Final prognosis of these patients is conditioned by the underlying ischemic cardiomyopathy and mechanical complications, such as mitral regurgitation or ventricular septal defect. For this reason, we strongly recommend to treat all concomitants heart diseases whenever possible, including coronary revascularization and mitral regurgitation.

\section{Founding}

This study was supported by Virgen de las Nieves Hospital and Ramón y Cajal Hospital. All surgical procedures 
described in the manuscript were realized using the material equipment and drugs support of these institutions.

\section{References}

[1] Hill, A.J., Laske, T.G., Coles, J.A., Sigg, D.C., Skadsberg, N.D., Vincent, S.A., Soule, C.L., Gallagher, W.J. and Iaizzo, P.A. (2005) In Vitro Studies of Human Hearts. The Annals of Thoracic Surgery, 79, 168-177. http://dx.doi.org/10.1016/j.athoracsur.2004.06.080

[2] Ballester-Rodés, M., Flotats, A., Torrent-Guasp, F., Carrió-Gasset, I., Ballester-Alomar, M., Carreras, F., Ferreira, A. and Narula, J. (2006) The Sequence of Regional Ventricular Motion. European Journal Cardio-Thoracic Surgery, 29, s139-s144. http://dx.doi.org/10.1016/j.ejcts.2006.02.058

[3] Vlodaver, Z., Coe, J.I. and Edwards, J.E. (1975) True and False Left Ventricular Aneurysm. Propensity for the Latter to Rupture. Circulation, 51, 567-572. http://dx.doi.org/10.1161/01.CIR.51.3.567

[4] Pretre, R., Linka, A., Jenni, R. and Turina, M.I. (2000) Surgical Treatment of Acquired Left Ventricular Pseudoaneurysms. The Annals of Thoracic Surgery, 70, 553-557. http://dx.doi.org/10.1016/S0003-4975(00)01412-0

[5] Atik, F., Navia, J.L., Ruda, P., Gonzalez-Stawinski, G.V., Alster, J.M., Gillinov, A.M., Svensson, L.G., Pettersson, B.G., Lytle, B.W. and Blackstone, E.H. (2007). Surgical Treatment of Postinfarction Left Ventricular Pseudoaneurysm. The Annals of Thoracic Surgery, 83, 526-531. http://dx.doi.org/10.1016/j.athoracsur.2006.06.080

[6] Frances, C., Romero, A. and Grady, D. (1998) Left Ventricular Pseudoaneurysm. Journal of the American College of Cardiology, 32, 557-561. http://dx.doi.org/10.1016/S0735-1097(98)00290-3

[7] Fujii, H., Hattori, R., Osako, M., Otani, H. and Imamura, H. (2001) Patch Repair of Postinfaction Pseudo-And Subepicardial Aneurysm of the Left Ventricle. The Journal of Cardiovascular Surgery, 42, 49-51. 